Published by Al-Nahrain College of Medicine P-ISSN 1681-6579

E-ISSN 2224-4719

Email: iraqijms@colmed-alnahrain.edu.iq

http://www.colmed-alnahrain.edu.iq

http://www.iraqijms.net

Iraqi JMS 2019; Vol. 17(1)

\title{
Incidence of Neurotrophic Keratopathy in Association with Diode Endolaser Retinal Photocoagulation
}

\author{
Najah k. M. Al-Quraishi ${ }^{1}$ FICS, H Dip Laser (Ophth), Abeer A. Mohammad ${ }^{2}$ FICS, Hind A. Mahdi ${ }^{2}$ \\ FICS
}

${ }^{1}$ Chairman of Iraqi Board in Ophthalmology, Baghdad, Iraq, ${ }^{2}$ Dept. of Surgery, Medical College, Al-Nahrain University, Baghdad, Iraq

\begin{abstract}
Background Neurotrophic keratopathy as a consequence of laser retinal photocoagulation is not uncommon problem but unfortunately and unfortunately it is not well evaluated in Iraq as well as the world.

Objective To estimate the incidence of neurotrophic keratophathy in association with diode endolaser retinal photocoagulation.

Methods This cross-sectional descriptive study was conducted in vitreoretinal clinic at Ibn-Alhaithum Teaching Eye Hospital in Baghdad. Patients were selected during a period from April 2013 to January 2014, all patients were examined by slit- lamp microscope, and condensing lens +78 Diopter for dilated fundus examination and cotton wisp to assess corneal sensation. Positive cases documented by anterior segment photograph.

Results The study sample consisted of 108 vitrectomizd eyes of 108 patients, their ages ranged between 5-65 years old with mean of ( 35.5 years), 42 of them were females and 66 of them were males. By follow up of those patients; 13 of their eyes (12\%) developed neurotrophic keratopathy. It had been found that $61.5 \%$ of those eyes manifested as stage II, while $30.8 \%$ presented as stage I and only $7.7 \%$ presented as stage III.

Conclusion Diode endolaser retinal photocoagulation may be an unrecognized reason for neurotrophic keratopathy

Keywords neurotrophic keratophathy, diode endolaser retinal photocoagulation, pars plana vitrectomy, diode laser $810 \mathrm{~nm}$

Citation Al-Quraishi NKM, Mohammad AA, Mahdi HA. Incidence of neurotrophic keratopathy in association with diode endolaser retinal photocoagulation. Iraqi JMS. 2019; 17(1): 18-23. doi: 10.22578/IJMS.17.1.4
\end{abstract}

List of abbreviations: $\mathrm{BIOM}=$ Binocular indirect ophthalmo microscope, $\mathrm{Ca}=\mathrm{Calcium} \mathrm{CO}_{2}=$ Carbonic dioxide, $\mathrm{MMPs}=$ Matrix metalloproteinases, $\mathrm{PPV}=$ Pars plana vitrectomy, $\mathrm{SDM}=\mathrm{Sub}$ visible diode micro pulse, $\mathrm{Zn}=\mathrm{Zinc}$

\section{Introduction}

The cornea is the principal refractive surface of the human eye and along with the sclera forms the outermost coat of the eyeball. It constitutes up to one-sixth of the entire eyeball. The corneal epithelium is derived from the surface ectoderm and the mesoderm gives rise to Bowman's layer, stroma, Descemet's membrane and endothelium. Tear film keeps the corneal surface moist and prevents the adherence of microbes. It also has many biologically active substances such as histamines, interleukins, prostaglandins and growth factors. Some of these factors modulate corneal epithelial migration, proliferation and differentiation ${ }^{(1)}$.

The cornea is primarily supplied by the sensory nerves derived from the ciliary nerves of the 
ophthalmic branch of the trigeminal nerve. The long ciliary nerves supply the perilimbal nerve ring. Nerve fibers penetrate the cornea in the deep peripheral stroma radially and then course anteriorly forming a terminal sub epithelial plexus. Presence of corneal sensation is vital to the maintenance of the integrity of the cornea. In cases of herpes simplex, herpes zoster and diabetes, corneal sensations are diminished and this may lead to persistent epithelial defects or delayed epithelial wound healing $(1,2)$.

The diode lasers emit a wavelength of $810 \mathrm{~nm}$ infrared in continuous wave mode. In the eye, diode laser light is absorbed only by melanin and consequently is most commonly used for retinal photocoagulation. Low scattering of this wavelength ensures good penetration of the ocular media and of edematous retina. The 810 $\mathrm{nm}$ wavelength also penetrates the sclera (3). Common clinical protocols use intra-operatively visible endpoints that cause iatrogenic chorioretinal damage. For this reason, laser therapy is normally limited to levels of disease severity for which the benefit-to-risk ratio justifies its application. The use of $810 \mathrm{~nm}$ diode laser in the Micro Pulse mode offers the surgeon the possibility to minimize iatrogenic retinal damage ${ }^{(4)}$.

\section{Pars plana vitrectomy (PPV)}

Small instruments, inserted through the pars plana, are used to cut and remove vitreous, peel membranes, and laser photocoagulate providing treatment to various posterior segment pathologies ${ }^{(5)}$.

\section{Neurotrophic keratitis}

Neurotrophic keratitis is a degenerative disease of corneal epithelium characterized by impaired healing. Absence of corneal sensitivity is the hallmark of the condition, which may end in corneal stromal melting and perforation. The causes of decreased corneal sensation are myriad and may affect sensory nerve supply from the trigeminal nucleus to the corneal nerve endings. Reduced corneal sensation renders the corneal surface prone to occult injury and decreases reflex tearing; it also appears to decrease healing rates of corneal epithelial injuries. Vulnerability and poor healing secondary to corneal sensory denervation favor the formation of nonhealing epithelial defects that tend to ulcerate and ultimately perforate if not appropriately treated in a timely fashion ${ }^{(6)}$.

\section{Clinical stages of neurotrophic keratitis}

Stage I: punctate epithelial staining with fluorescein

Stage II: Acute loss of epithelium

Stage III: Stromal lysis

This study was conducted to estimate the incidence of neurotrophic keratophathy in association with diode endolaser retinal photocoagulation.

\section{Methods}

This cross-sectional descriptive study was conducted in Ibn-Alhaithum Teaching Hospital, the Vitreoretinal Clinic, from April 2013 to January 2014. A one hundred eight sample of patients received diode laser retinal photocoagulation during pars plana vitrectomy done by three vitreoretinal surgeons. The patients who were enrolled in this study include all vitrectomizd patients who were attending the vitreoretinal clinic of the three vitreoretinal surgeons.

\section{Exclusion criteria}

Patients with diabetes, corneal surgery, herpetic eye disease, chronic glaucoma medication, patients with pars plana vitrectomy but without endolaser and age above 65 years (old age group).

\section{Collection of data}

Data were collected using a pre-constructed data collection form, which was formulated for purpose of this study and it was validated by the supervisor. The data collected included:
1. Name
2. Age
3. Gender
4. History of diabetes
5. Previous corneal surgery 
6. History of herpetic infection

7. History of chronic drug use (especially glaucoma medication)

8. History of contact lens use

9. Duration of operation

10. Scraping of corneal epithelium

11. Pattern of laser (localized or 360 degree)

12. Visual acuity

13. Treatment

The followings were taken from the case sheet of the patients:

Name of senior

Reference number

Date of operation

All patients were examined using slit- lamp microscope, condensing lens +78 diopter for dilated fundus examination and cotton wisp to assess corneal sensation. Positive cases documented by anterior segment photograph.

\section{Ethical issue}

This research has been approved by scientific council of ophthalmology.

Verbal consent has been obtained from the patients.

\section{Statistical analysis}

Statistical analysis was conducted by using Excel Microsoft Office 2013.

\section{Results}

The study sample consisted of 108 vitrectomizd eyes of 108 patients, their ages ranged between 5-65 years old with mean of (35.5 years), 42 of them were related to females and 66 were related to males as shown in table 1.

Table 1. Baseline characteristics of study group

\begin{tabular}{ccc}
\hline Gender & Number & Percentage \\
\hline Female & 42 & 38.8 \\
Male & 66 & 61.2 \\
\hline Total & 108 & 100 \\
\hline
\end{tabular}

The higher incidence of neurotrophic keratopathy occurs among age group range from 25 years to less than 35 years as shown in table 2.

By follow up of those patients; 13 of their eyes (12\%) develops neurotrophic keratopathy as shown in figure 1.
It had been found that $61.5 \%$ of those eyes manifested as stage II, while $30.8 \%$ presented as stage I and only $7.7 \%$ presented as stage III as shown in table 3.

Table 2. The incidence of neurotrophic keratopathy among different age groups

\begin{tabular}{ccc}
\hline Age (years) & Frequency & Percentage \\
\hline $5-14$ & 2 & 15.39 \\
$15-24$ & 1 & 7.69 \\
$25-34$ & 5 & 38.47 \\
$35-44$ & 2 & 15.39 \\
$45-54$ & 2 & 15.39 \\
$55-64$ & 1 & 7.69 \\
\hline
\end{tabular}




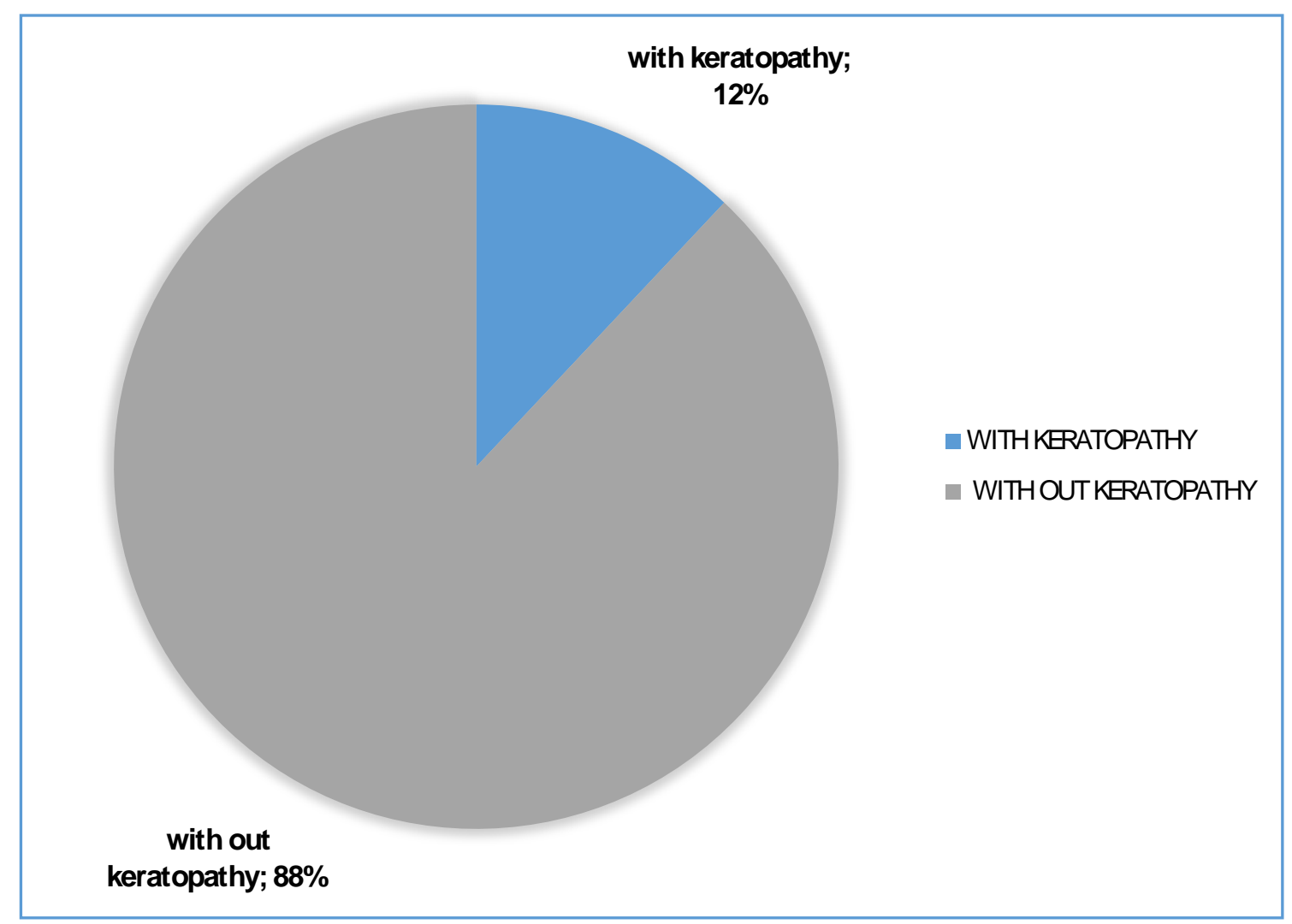

Figure 1. Incidence of neurotrophic keratopathy in association with diode endolaser retinal photocoagulation

Table 3. Frequency distribution and percentage of clinical stage

\begin{tabular}{ccc}
\hline Clinical stage & Number of eyes & Percentage \\
\hline Stage $1^{*}$ & 4 & 30.8 \\
Stage $2^{* *}$ & 8 & 61.5 \\
Stage $3 * *$ & 1 & 7.7 \\
\hline Total & 13 & 100 \\
\hline
\end{tabular}

* stage I: Punctate epithelial staining with fluorescein

** stage II: Epithelial defect

$* * *$ stage III: Stromal lysis, sometimes resulting in corneal perforation

\section{Discussion}

Neurotrophic keratitis is a degenerative disease of corneal epithelium characterized by impaired healing. Absence of corneal sensitivity is the hallmark of the condition. The causes of decreased corneal sensation are myriad and may affect sensory nerve supply from the trigeminal nucleus to the corneal nerve endings
(7). One of the iatrogenic causes of neurotrophic keratophathy is trauma to ciliary nerves by laser $(1,6,8)$.

The diode lasers emit a wavelength of $810 \mathrm{~nm}$ infrared in continuous wave mode. Low scattering of this wavelength ensures good penetration of the ocular media and of edematous retina. The $810 \mathrm{~nm}$ wavelength also 
penetrates the sclera ${ }^{(4)}$. It is of high power and deeper penetration into the choroids ${ }^{(9)}$. Sub visible diode micro pulse (SDM) is applied for non-damaging retinal phototherapy by using shorter bursts with small spot size (100-200 micrometer) ${ }^{(10)}$.

In the present study, 108 patients; who had undergone pars plana vitrectomy with diode endolaser retinal photocoagulation; 13 (12\%) of them developed neurotrophic keratopathy. Other causes of neurotrophic keratopathy were excluded to illustrate the association with diode endolaser retinal photocoagulation. Laser retinal photocoagulation well known to cause this condition and the diode laser specifically has physical ability to be absorbed by the fundus to somewhat unpredictable way ${ }^{(10)}$. Diode laser pan retinal photocoagulation associated with neurotrophic keratopathy especially while using it in high power ${ }^{(11)}$.

Unfortunately, there are no studies similar to this study to compare with. Recently, two studies were published that support this study. Banerjee et al report a series of 5 cases of patients without diabetes who developed neuropathic corneal ulceration presumed secondary to long ciliary nerve compromise following vitrectomy surgery with endolaser and silicone oil tamponade for retinal detachment ${ }^{(12)}$. Doyle et al found that pars plana vitrectomy with pan retinal photocoagulation may be an unrecognized cause of neurotrophic keratopathy ${ }^{(13)}$.

So, the question that could be answered is that the diode endolaser retinal photocoagulation while used in continuous mode may be the cause of neurotrophic keratopathy.

The limitations of the study are:

- Small sample size

- The examination of corneal sensation was not done by esthesiometer as it is not available which is more reliable and give quantitative results.

- There was no documentation in the case sheet of all patients for epithelial scraping which is needed in some patients intraoperatively, so it can't be correlated as risk factor for neurotrophic keratopathy.
- Also the number of laser shoots and the power used not documented in the case sheets.

- Follow up period is not the same for all patients.

This study concluded that diode endolaser retinal photocoagulation may be an unrecognized reason for neurotrophic keratopathy.

The recommendations of this study are:

- Studying of a larger sample size

- Studying of two groups, one with epithelial scraping and other without.

- Studying of two groups, one treated with diode laser $810 \mathrm{~nm}$ and the other group should be treated with less penetrating photocoagulater laser e.g. Nd:YAG laser 530 $\mathrm{nm}$.

- Also using high power density laser blamed for appearance of complications, so make sure to use as minimum as possible power density especially at 3.00 and 9.00 o'clock of the retinal periphery.

- SDM is showing promise as it is applied for non-damaging retinal phototherapy by using shorter bursts with small spot size (100-200 micrometer).

- Documentation of epithelial scraping and number of laser shoots and the power used in the case sheets.

\section{Acknowledgments}

The author would like to express his gratitude to his supervisor Assistant Prof. Dr. Najah AlQuraishi for his great assistance, kind advice \& scientific guidance. Also, to thank Assistant Prof. Dr. Faiz Al-shakarchi for his kind support, Dr. Ali Al-Fazaa for his help in data collection. Also, the author is grateful to the staff of statistics unit of the Ibn AL Haitham teaching eye hospital.

\section{Author contribution}

All authors have contributed equally to this article.

\section{Conflict of interest}

The authors declare no conflict of interest.

Funding

None. 


\section{References}

1. Sharma N, Vajpayee RB. Corneal ulcers diagnosis and management. New Delhi: Jaypee Brothers Medical Publishers Ltd; 2008. p. 3-5, 16, 154-61.

2. Remington LA, Goodwin D. Clinical anatomy and physiology of the visual system. $3^{\text {rd }}$ ed. Missouri: Elsevier Butter worth Heinemann; 2012. p. 219.

3. Elkington AR, Frank HJ, Greaney MJ. Clinical optics. 3rd ed. UK: Blackwell Science; 1999. p. 221-4.

4. Dorin G. Subthreshold and micropulse diode laser photocoagulation. Sem Ophthalmol. 2003; 18(3), 14753.

doi: https://doi.org/10.1076/soph.18.3.147.29812.

5. Bernardino $C R$. Yale guide to ophthalmic surgery. Lippincott Williams \& Wilkins; 2011. p. 153-4.

6. Krachmer JH, Mannis MJ, Holland EJ. Cornea fundamental, diagnosis and manegment. $3^{\text {rd }}$ ed. MOSBY Elsevier; 2011. p. 90.

7. Bhagat $N$, Zarbin MA. Subthreshold micropulse diode laser for DME. Retinal Physician. 2011 (accessed 3 May 2014).

8. Davis EA, Dohlman $\mathrm{CH}$. Neurotrophic Keratitis. Int Ophthalmol Clin. 2001; 41(1): 1-11.

9. Bhattacharyya B. Step by step laser in ophthalmology. New Delhi: Jaypee Brothers Medical Publishers Ltd; 2009. p. 8.

10. Garge A, Lin JT, Aloi JL, et al. Mastering the technique of LASER application in ophthalmology. New Delhi: Jaypee Brothers Medical Publishers; 2008. p. 476, 744.
11. Bandello F, Brancato $R$, Trabucchi $G$, et al. Diode versus argon-green laser panretinal photocoagulation in proliferative diabetic retinopathy: a randomized study in 44 eyes with a long follow-up time. Graefes Arch Clin Exp Ophthalmol. 1993; 231(9): 491-4. doi: 10.1007/BF00921112.

12. Banerjee PJ, Chandra A, Sullivan PM, et al. Neurotrophic corneal ulceration after retinal detachment surgery with retinectomy and endolaser: a case series. JAMA Ophthalmol. 2014; 132(6): 750-2. doi: 10.1001/jamaophthalmol.2014.280.

13. Doyle JI, Hall T, Michelson MA. Neurotrophic keratopathy following pars plana vitrectomy and indirect ophthalmoscopic laser. ASCRS.ASOA Boston Symposium and Congress; 2014.

\section{Correspondence to Dr. Hind A. Mahdi E-mail: H.daffodel@gmail.com aberamir@colmed-alnahrain.edu.iq Received Jun. $6^{\text {th }} 2018$ Accepted Dec. 18 2018}

